\title{
Prevalencia de Caries, Pérdida de Dientes y Necesidad de Tratamiento en Población Adulta Mapuche-Huilliche de Isla Huapi
}

\author{
Prevalence of Caries, Teeth Loss and Treatment Needs in Adult \\ Mapuche-Huilliche Population in Isla Huapi
}

Angel $\mathrm{P}^{1}$, Fresno $\mathrm{MC}^{1}$, Cisternas $\mathrm{P}^{1}$, Lagos $\mathrm{M}^{2}$, Moncada $\mathrm{G}^{3}$

\begin{abstract}
RESUMEN
Objetivo: El objetivo de este estudio transversal fue evaluar el estado de salud oral y cuantificar la prevalencia de caries dental, pérdida de dientes y necesidad de tratamiento en la población adulta de etnia Mapuche-Huilliche que habita la Isla Huapi. Método: Se examinó una muestra representativa de 64 habitantes adultos de la Isla Huapi, constituida por 31 hombres y 33 mujeres de entre 25 a 82 años de edad, que tenían a lo menos un apellido de origen Mapuche-Huilliche. Todos los individuos, previa firma de un consentimiento informado, fueron examinados por un solo dentista calibrado durante el mes de agosto 2009. Los datos fueron registrados en una ficha diseñada especialmente y analizados estadísticamente usando los test T de Student y ANOVA. Resultados: El promedio del índice COPD de la muestra fue 14.59. Todos los individuos tenían historia de caries. De los que conservaban dientes, el $71.43 \%$ tenían caries sin tratar, con un promedio de 1.25 . El $98.43 \%$ de los individuos de la muestra presentaban algún grado de desdentamiento, el $12.5 \%$ eran edéntulos. El 100\% de la población necesitaba atención odontológica, ya fuera para operatoria o prótesis. Conclusiones: Esta población presenta un gran daño en cuanto a su salud oral, especialmente debido a pérdida de dientes. La magnitud del daño e importancia del entorno social como determinante en la generación de éste, obliga a enfrentar el problema y buscar soluciones desarrollando y aplicando programas gubernamentales de promoción y atención en salud oral a toda la población.
\end{abstract}

Rev. Clin. Periodoncia Implantol. Rehabil. Oral Vol. 3(2); 69-72, 2010.

Palabras clave: Prevalencia, caries, edentulismo, etnia Mapuche, adultos.

\section{ABSTRACT}

Objectives: The aim of this cross- sectional study was to assess oral health status and quantify prevalence of dental caries, teeth loss and treatment needs in an ethnic group of Mapuche-Huilliche adult population in Isla Huapi. Method: A representative sample of 64 adult inhabitants of Isla Huapi was selected. 31 men and 33 women aged 25 to 82 years of age who had at least one Mapuche-Huilliche surname, after signing an informed consent, individuals were examined by a calibrated examiner during August 2009. Data were recorded and analyzed statistically using T Student and ANOVA tests. Results: The DMFT index average of the sample was 14.59. All individuals had caries experience. Untreated caries were found in a $71.43 \%$ of individuals retaining teeth, with an average of 1.25 each. $98.43 \%$ of the individuals had some degree of edentulism, $12.5 \%$ were edentulous. $100 \%$ of the population needed either restorative or prosthetic dental care. Conclusions: This population has a high degree of damage in their oral health, especially due to teeth loss, which could be explained by their limited access to preventive and restorative dental care. The extent of the damage and the importance of the social environment as a determiner in the generation of it, will mean addressing the problem and seeking solutions by developing and implementing government programs for the promotion and oral health care to the entire population.

Rev. Clin. Periodoncia Implantol. Rehabil. Oral Vol. 3(2); 69-72, 2010.

Key words: Prevalence, caries, edentulism, Mapuche ethnic group, adults.

\section{INTRODUCCIÓN}

La caries dental es una enfermedad de alta prevalencia y severidad en las poblaciones de diversos países. En los países industrializados afecta a más de la mitad de la población, y por ser un proceso acumulativo, la severidad del daño se incrementa a medida que aumenta la edad ${ }^{(1,2,3)}$.

Desde la perspectiva de causalidad, la caries es una enfermedad compleja ya que es originada por la interacción de distintos mecanismos. Para su análisis se deben considerar la acción de varios genes, factores ambientales, culturales, sociales y locales ${ }^{(4)}$.

El fortalecimiento en el enfoque social de los determinantes de salud ha dado mayor importancia al entorno social, tanto en la determinación del estado de salud oral de la población como en la respuesta en términos de intervenciones sanitarias, en lugar de enfocarse sólo en cambios del comportamiento individual|(5,6).

La información sobre el estado de salud oral es esencial para las decisiones gubernamentales en los programas de cuidado y prevención de ésta. Actualmente en Chile existen escasos estudios de la prevalencia de caries dental en la población Mapuche ${ }^{(7)}$, desconociéndose las necesidades de tratamiento, así como tampoco los recursos humanos necesarios para resolver las secuelas de esta patología.

El pueblo Mapuche se distribuye en diversas comunidades indígenas, una de las cuales se encuentra en Isla Huapi, ubicada en el Lago Ranco, sur de Chile. Por su ubicación y la inexistencia de rutas de comunicación terrestres, la comunidad de Isla Huapi es considerada un semi-aislado geográfico.

El objetivo de este trabajo fue determinar la prevalencia de lesiones de caries dental, dientes obturados por caries y pérdida de diente en adultos de etnia Mapuche-Huilliche de edades sobre 25 años que habitan en Isla Huapi y determinar las necesidades reales de tratamiento para esta población.

1. Instructor. Departamento de Odontología Restauradora, Área Operatoria Dental. Facultad de Odontología, Universidad de Chile. Chile.

2. Cirujano Dentista. Universidad de Chile. Chile.

3. Prof. Titular. Departamento de Odontología Restauradora, Área Operatoria Dental. Facultad de Odontología, Universidad de Chile. Chile.

Correspondencia autor: M. Consuelo Fresno Rivas. consue_fresno@yahoo.com. Trabajo recibido el 20/04/2010. Aprobado para su publicación el 30/08/2010. 


\section{MATERIAL Y MÉTODO}

El lugar de estudio correspondió a la Isla Huapi, localizada en la provincia de Valdivia, Región de los Ríos, Chile. En ésta viven 390 personas de origen Mapuche-Huilliche, según el censo de 2002, cuyos índices de indigencia son los mas altos a nivel provincial(8).

Del total de 224 adultos sobre 25 años con al menos un apellido Mapuche-Huilliche se realizó una muestra aleatoria simple. Para determinar el tamaño de la muestra se usó un nivel de confianza del $90 \%$, estimándose que el $71.4 \%$ de población estaba afectada con al menos una caries y un error de estimación de $9.3 \%$, se obtuvo así un tamaño muestral de 64 individuos.

Una vez obtenidos los mapas con la ubicación de la casas, proporcionados por el director del colegio de Isla Huapi, se seleccionó aleatoriamente las casas a visitar procediéndose a realizar viajes por vía terrestre durante dos semanas hasta completar la muestra.

Los individuos fueron evaluados clínicamente por un sólo operador calibrado de acuerdo a los criterios de la OMS para índice COPD ${ }^{(9)}$. El operador fue entrenado por un clínico con experiencia, examinando un grupo de 20 pacientes en dos oportunidades con dos semanas de diferencia, aprobando la evaluación cuantitativa de confiabilidad intraexaminador con un índice Kappa 0.966.

Los 64 participantes de este estudio aceptaron libremente participar mediante un consentimiento informado.

Los exámenes fueron realizados en una silla en cada casa de los examinados, utilizando espejo bucal número 5 , pera de aire para secar las superficies dentales y luz artificial. Antes del examen y cuando correspondía, se procedió a la remoción de la o las prótesis dentales.

Los datos obtenidos fueron registrados en una ficha especialmente diseñada para ello.

Se hizo un análisis descriptivo de frecuencias con valores absolutos y porcentajes para las variables categóricas y medidas de dispersión, tendencia central para las variables continuas tales como edad e índice COPD.

La significancia estadística se consideró cuando $p<0.05$. Se ajustó un modelo de regresión lineal donde el COPD era la variable dependiente y considerando la variable edad como variable independiente.

Para realizar los análisis estadísticos se utilizó el test T de Student y ANOVA, usando el programa estadístico SPSS 15.0.

\section{RESULTADOS}

La muestra quedó constituida por $33(51.56 \%)$ mujeres y 31 (48.43\%) hombres.

El promedio de edad fue 44.54 años, siendo mayor el de los hombres (48.51 años) que el de las mujeres $(40.81)(p=0.02)$ (Tabla 1).

\section{Prevalencia y Severidad de Caries}

El $100 \%$ de los individuos presentó historia de caries. Para el total de la muestra, el promedio del índice COPD fue de 14.59 con un intervalo de confianza (IC) de 12.61 y 16.57 (Tabla 2).

Este valor estuvo determinado principalmente por el número de dientes perdidos por caries $(P)$, con una media de 13.07 con un IC de 10.92 y 15.23. Para el componente piezas dentarias obturadas $(O)$ se encontró una media de 0.26 con un IC de 0.02 y 0.51 . Para el componente caries (C) se observó una media de 1.25 con IC de 0.94 y 1.48 (Gráfico 1).

Tabla 1. Promedio de edad por género de los individuos.

\begin{tabular}{|l|c|c|c|c|}
\hline Sexo & $\boldsymbol{u}$ & Media (años) & Error estándar & Intervalo de confianza (95\%) \\
\hline Mujer & 33 & 40.81 & 12.77 & $36.28-45.34$ \\
\hline Hombre & 31 & 48.51 & 13.10 & $43.70-53.32$ \\
\hline Total & 64 & 44.54 & 13.40 & $41.19-47.89$ \\
\hline
\end{tabular}

Tabla 2. Índice COPD para toda la muestra.

\begin{tabular}{|l|c|c|c|c|}
\hline Índice & $\boldsymbol{u}$ & Media & Error estándar & Intervalo de confianza (95\%) \\
\hline $\mathrm{C}$ & \multirow{3}{*}{64} & 1.25 & 1.43 & $0.89-1.60$ \\
\cline { 4 - 5 } & 0.26 & 0.97 & $0.02-0.51$ \\
\cline { 3 - 5 } $\mathrm{P}$ & 13.07 & 8.61 & $10.92-15.23$ \\
\cline { 3 - 5 } $\mathrm{COPD}$ & & 14.59 & 7.91 & $12.61-16.57$ \\
\hline
\end{tabular}

Gráfico 1. Índice COPD. El Gráfico 1 muestra que el índice COPD de toda la muestra es 14.59, está influenciado principalmente por la pérdida de dientes $(\mathrm{P}) 13.07$.

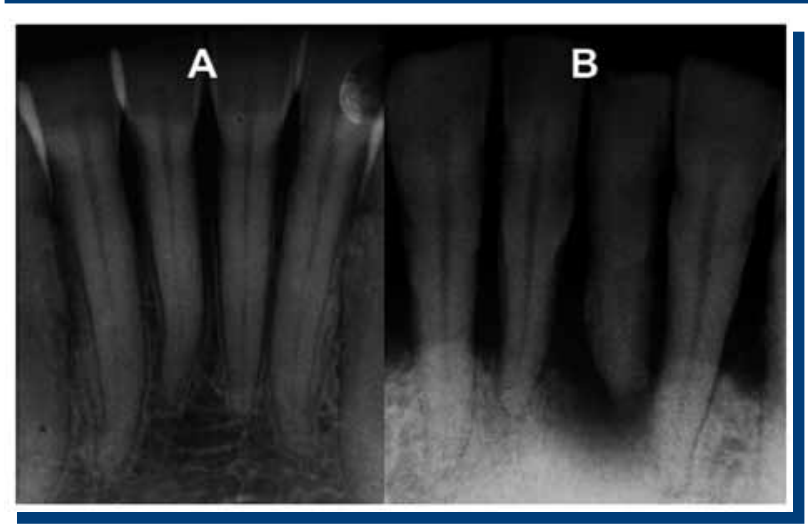

El COPD según género no mostró diferencias significativas $(p=0.391)$, con un valor de 14.66 (IC 12.04-17.28) para mujeres y 14.51 (IC 11.37-17.65) para hombres.

El promedio de lesiones de caries para mujeres fue 1.15 (IC $0.61-168)$ y para hombres 1.35 (IC $0.84-1.86$ ), no hubo diferencias estadísticamente significativas por género $(p=0.867)$.

En el promedio de dientes obturados por caries, a pesar de no existir diferencias estadísticamente significativas $(p=0.184)$ según género, el valor fue mayor para las mujeres 0.42 (IC 0.04-0.89) que para hombres 0.09 (IC 0.01-0.20).

El promedio del número de dientes perdidos para las mujeres fue de 13.09 (IC 10.18-15.99), para los hombres fue 13.06 (IC 9.69$16.43)$, sin diferencias estadísticamente significativas $(p=0.452)$.

\section{Prevalencia de Edentulismo y Uso de Prótesis}

De la muestra el $98.43 \%$ tuvo pérdida de dientes, con un rango entre 1 y 28 . Sólo una persona conservaba su fórmula dentaria completa.

En esta muestra ocho personas eran edéntulas (12.5\%), de los cuales $62.5 \%$ correspondió a hombres y $37.5 \%$ a mujeres.

El $87.5 \%$ de la muestra fueron desdentados parciales, de los cuales el $43.63 \%$ fueron desdentados parciales bimaxilares (Tabla 3 ).

Tabla 3. Desdentados parciales según maxilar.

\begin{tabular}{|l|c|c|c|}
\hline Condición & $\boldsymbol{u}$ & Proporción & Intervalo de confianza (95\%) \\
\hline Desdentado Parcial Maxilar & 40 & $62.5 \%$ & $47.49 \%-77.50 \%$ \\
\hline Desdentado Parcial Mandibular & 33 & $51.56 \%$ & $34.51 \%-68.61 \%$ \\
\hline $\begin{array}{l}\text { Desdentado Parcial Ambos } \\
\text { Maxilares }\end{array}$ & 24 & $37.5 \%$ & $18.13 \%-56.86 \%$ \\
\hline
\end{tabular}

De los pacientes desdentados totales bimaxilares, el $50 \%$ era portador de prótesis superior e inferior. De un total de 12 individuos desdentados totales superiores y parciales inferiores, ocho (66.66\%) usa prótesis superior y ninguno de ellos usa prótesis parcial inferior

\section{Determinación de Recursos Humanos Requeridos (Dentistas y Laboratoristas Dentales)}

\section{a) Recursos humanos necesarios para tratar lesiones de caries}

El promedio de dientes cariados por individuo fue de 1.25 , es decir, la muestra presenta 80 dientes sin tratar pero si el índice $C 1.25$, se multiplica por el total de la población adulta sobre 25 años (224), da un total de 280 dientes que necesitan tratamiento por lesiones de caries.

Aplicando el rendimiento de obturación por hora profesional odontológica según las normas del Ministerio de Salud, que indican como rendimiento mínimo 1.12 dientes obturados por hora, se necesitarían 250 horas odontológicas para tratar las lesiones de caries existentes en los adultos de la isla, por lo tanto se necesitaría 1 dentista trabajando 8 horas diaria por 31.25 días.

b) Recursos humanos necesarios para efectuar prótesis removibles de 75 (Tabla 4) 
Tabla 4. Necesidad de prótesis por grupo etáreo y género.

\begin{tabular}{|l|c|c|c|}
\hline \multirow{2}{*}{ Grupo Etáreo } & \multicolumn{2}{|c|}{ Género } & \multirow{2}{*}{ Total } \\
\cline { 2 - 3 } & Masculino & Femenino & 32 \\
\hline 25 - 44 años & 11 & 21 & 34 \\
\hline 45 - 64 años & 17 & 17 & 9 \\
\hline 65 y más años & 6 & 3 & 75 \\
\hline Total & 34 & 41 & \\
\hline
\end{tabular}

Aplicando el rendimiento de prótesis removible de acuerdo a las normas del Ministerio de Salud, que indican como rendimiento mínimo de 0.35 prótesis por hora, es decir, 2.85 horas por prótesis como tiempo mínimo, se necesitarían 213.75 horas para efectuar las prótesis, esto significa 1 dentista trabajando una jornada completa de 8 horas durante 26.71 días para solucionar los problemas de desdentamiento de la población estudiada.

Si se considera el número de prótesis a confeccionar, 75 , y se aplica el rendimiento para laboratoristas dentales según las normas del Ministerio de Salud de Chile, que indican como tiempo mínimo 4 horas por prótesis, se necesitarían un total de 300 horas laboratorista dental, esto significa un laboratorista trabajando 8 horas diarias por 37.5 días.

Al extrapolar estos resultados a toda la población adulta de Isla Huapi, 224 individuos, el número de prótesis a confeccionar sería 262.5 , esto significa 748.125 horas odontológicas, es decir, un dentista a jornada completa por 93.52 días y un laboratorista dental, 1.050 horas, 131.25 días laborales en las mismas condiciones para rehabilitarlos con prótesis removibles.

\section{DISCUSIÓN}

Este estudio fue realizado en una población adulta sobre 25 años, representantes de la cultura Mapuche-Huilliche de una área rural, semi aislada y con acceso limitado a atención odontológica, ya que la posta sólo recibe ronda médico dental una vez al mes. Debido a su singular situación geográfica la Isla Huapi, ubicada en el sur de Chile, provincia de Valdivia, Región de los Ríos, presenta la mayor concentración de esta etnia aislada geográficamente del país, de la cual se desconocen indicadores de salud oral.

Este estudio proporciona una base de datos útiles acerca de la prevalencia y severidad de caries, estableciendo las necesidades de tratamiento en dicho grupo poblacional perteneciente a una minoría étnica rural aislada y con acceso limitado a la atención odontológica.

La prevalencia de caries encontrada en la población fue del $100 \%$ con una gran severidad, expresada por un alto índice de desdentamiento. Esto se observa en el índice COPD cuyo componente $\mathrm{P}(13.07)$ representa el $90 \%$.

Los resultados de este estudio muestran que a medida que aumenta la edad de los individuos, el índice COPD es mayor, siendo los dientes perdidos por caries dental la principal causa de estos altos índices, como lo muestra Fure y cols. ${ }^{(10,11)}$, en un estudio de seguimiento a una población de 55 a 75 años de edad de Gotegord, Suecia por un periodo de 10 años.

El índice COPD para la muestra total fue de 14.59 cuyos componentes son 1.25 dientes con lesiones de caries cavitadas, 0.26 dientes obturados sin caries y de 13.07 dientes perdidos por caries. El COPD del grupo etario de 25 a 44 años es de 9.36 (Gráfico 2); el de 45 a 64, 19.72 (Gráfico 3), y mayores de 65 fue de 27.17 (Gráfico 4). Este resultado muestra el gran daño acumulado a través de los años producto de la caries dental en la población en estudio. Lo que concuerda con los estudios de Caplan y cols. ${ }^{(10)}$, White y cols. ${ }^{(11)}$, Fure ${ }^{(12)}$, Fure y Zicker ${ }^{(13)}$, Wang y cols. ${ }^{(14)}$, Madlena y cols. ${ }^{(15)}$, Gamonal(16), Urzúa(17), Rihs y cols. ${ }^{(18)}$.
Gráfico 2. Índice COPD 25 a 44 años. El Gráfico 2 muestra que el índice COPD de 9.36 está influenciado principalmente por la pérdida de dientes $(P) 7.44$.

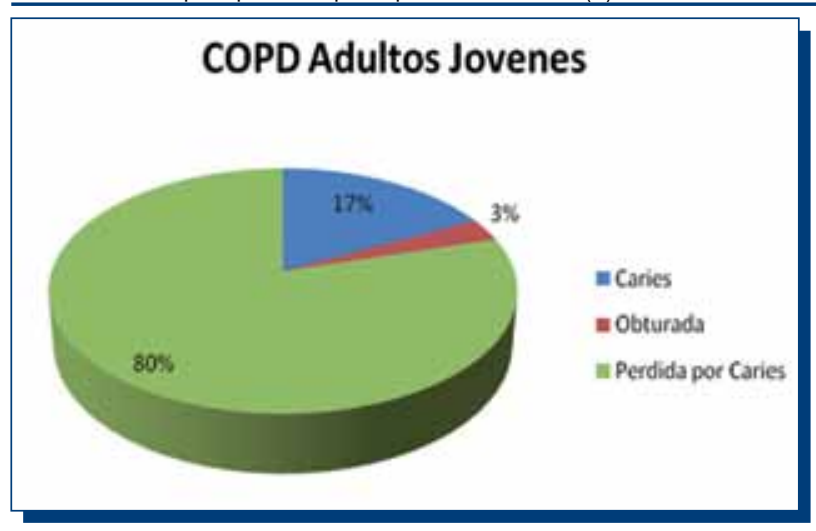

Gráfico 3. Índice COPD 45 a 64 años. El Gráfico 3 muestra que el índice COPD aumenta en este grupo etáreo a 19.72 y está influenciado principalmente por la pérdida de dientes $(P)$ 18.68.

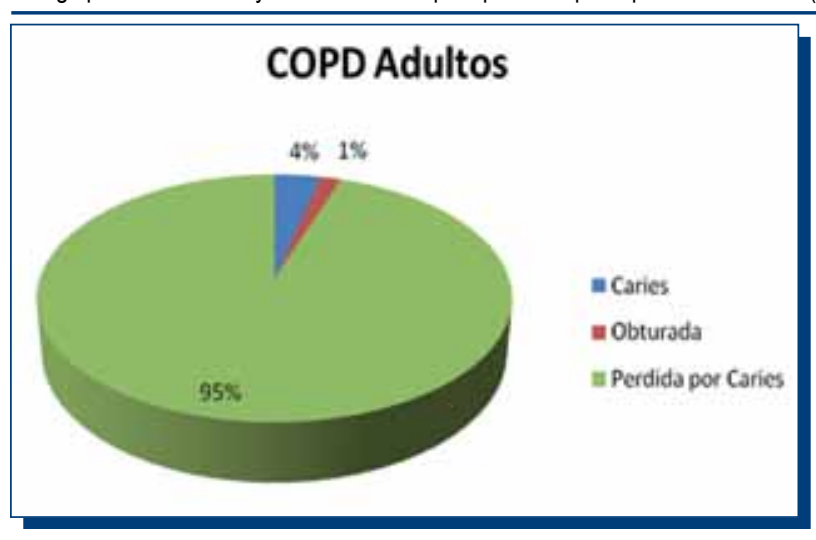

Gráfico 4. Índice COPD 45 a 64 años. El Gráfico 4 muestra que el índice COPD de los adultos mayores ha aumentado a 27.17 y también está influenciado principalmente por la pérdida de dientes $(\mathrm{P}) 26.33$

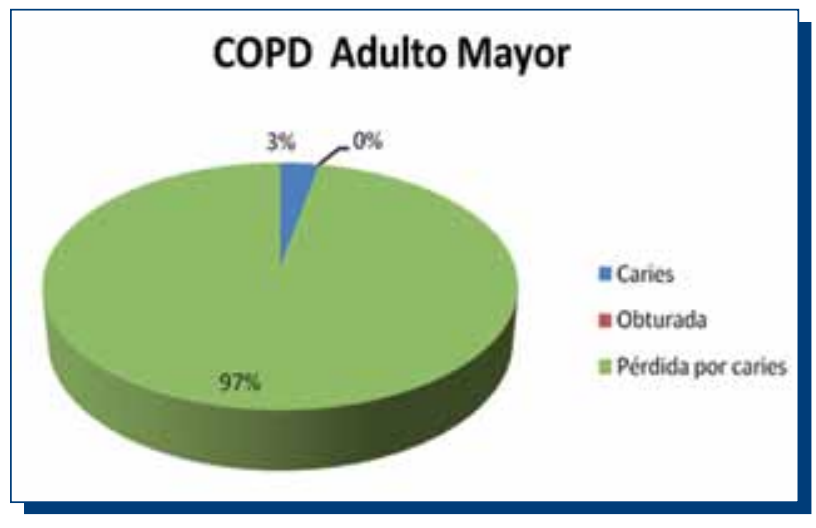

Las mujeres presentaron mayor cantidad de dientes obturados que los hombres, pero no se encontró diferencia estadística en el número de obturaciones por sexo. Este número de obturaciones es significativamente menor que en países desarrollados como Inglaterra, con un promedio de dientes obturados de 11.1, en Escocia de 10.7 y en Reino de Países Bajos de 10.6 ${ }^{(19,20)}$, lo que podría deberse a una falta de atención odontológica oportuna para tratamientos conservadores.

El promedio de lesiones de caries cavitadas por individuo encontrado en este estudio fue de 1.25 y a pesar de que no existen diferencia estadística por sexo ( $p=0.867)$, el número de lesiones de caries fue mayor en hombres (1.35) que en mujeres (1.15), situación similar a lo reportado por Brennan y Spencer ${ }^{(21)}$ en pacientes australianos.

El promedio de dientes perdidos por caries por individuo encontrado en este estudio es de 13.01. Al ser una población de estrato socioeconómico bajo es probable que este valor sea resultado de una falta de tratamientos conservadores y preventivos. En Chile, la atención odontológica es una de las áreas, junto a salud mental, en las que existen las mayores brechas entre oferta y necesidades. Como ya se 
ha señalado, aquellos grupos con mayor necesidad de atención y menor acceso sólo estarían recibiendo atención de urgencia, lo que finalmente podría terminar en una mayor pérdida de piezas dentarias.

De los 64 individuos que constituyeron este estudio, el $12.5 \%$ eran desdentados totales ambos maxilares. El $32.81 \%$ eran desdentados totales del maxilar superior y el $14.06 \%$ desdentados totales inferiores. Bourgeois y cols. ${ }^{(22)}$ muestran cifras que van de $12.8 \%$ a $69.6 \%$ de desdentados para los países europeos.

Las posibles razones que explican el gran daño encontrado en la población de Isla Huapi podrían ser que a medida que avanza la edad en un individuo se presentan algunos indicadores de riesgo de caries, como son: higiene oral defectuosa debido a los problemas motrices inherentes a la edad ${ }^{(23)}$, también en este caso, falta de educación en salud oral, de implementos necesarios para el cuidado de ella sumado a una baja frecuencia de control y atención odontológicos debido al aislamiento geográfico y condiciones climáticas adversas que impiden un mejor acceso a centros de salud.

Según los datos de la Primera Encuesta Nacional de Salud en Chile realizada el año 2003 , el $33.4 \%$ de la población mayor a 65 años de edad no presenta ninguna pieza dentaria en boca ${ }^{(24)}$. Este valor es más alto que el encontrado en este estudio. La razón de esto podría ser que en la cifra reportada en la Encuesta Nacional de Salud esta el promedio nacional, el cual incluye zonas geográficas distintas así como áreas rurales y urbanas.

La solución más racional para lograr una mejoría en la salud bucal de las futuras generaciones, especialmente en las poblaciones indígenas, sería la implementación de medidas de promoción, prevención y control de lesiones de caries, las que muestran su efectividad en algunas poblaciones de países desarrollados al anticiparse a la enfermedad y de esa forma permiten optimizar los recursos humanos y materiales presentando una relación costo-beneficio muy favorable.
La magnitud de la brecha y la importancia del entorno social en la generación del daño de la salud oral, obliga a enfrentar el problema desde una perspectiva amplia de determinantes sociales de la salud, paradigma que nos orienta en un cambio en la promoción de salud oral, lo que se traduce en que sin abandonar los esfuerzos por mejorar la equidad de acceso a la atención, las intervenciones sanitarias que se implementen consideren el entorno social y geográfico en lugar de enfocarse sólo en cambios del comportamiento individual.

\section{CONCLUSIONES}

A la luz de los resultados obtenidos se puede concluir que existe un daño enorme en la salud oral de esta población con un $100 \%$ de prevalencia de caries en aquellos individuos con dientes remanentes. El índice COPD para el total de la muestra es 14.59 , y se ve incrementado significativamente con la edad, sin diferencias por género.

Los hombres presentan mas dientes cariados y perdidos por caries que las mujeres. A mayor edad existe un mayor daño representado en un aumento considerable del índice COPD en todos sus componentes. El componente $\mathrm{P}$ es el que contribuye en mayor proporción a este índice en todos los grupos etáreos y determina el alto grado de desdentamiento en la población. Todos los individuos examinados necesitan atención odontológica, ya sea en procedimientos restauradores como en tratamientos protésicos.

Se espera que los resultados entregados en el presente estudio sean útiles a las autoridades sanitarias, haciéndolos concientes de esta realidad y así sirvan para el diseño, implementación y desarrollo de programas odontológicos destinados prioritariamente a los grupos más vulnerables.

\section{REFERENCIAS BIBLIOGRÁFICAS}

1. Peterson HG, Bratthall D. The caries decline: a review of reviews. Eur J Oral Sci,1996;104:436-443.

2. Burt B. Concepts of risk in dental public health. Community Dent Oral Epidemiol,2005;33(4):240-7.

3. Griffin SO, Griff PM, Swann JL, Zlobin N. New coronal caries in older adults: impications for prevencion. J Dent Res,2005;84(7):715-720.

4. Fejerskov $O$. Concepts of dental caries and their consequences for understanding the disease. Community Dent Oral Epidemiol,1997;25:5-12. 5. Sabbah W, Tsakos G, Chandola T, Sheiham A, Watt RG. Social gradients in oral and general health. J Dent Res,2007;86(10):992-6.

6 . Watt RG. Emerging theories into the social determinants of health: implications for oral health promotion. Review. Community Dent Oral Epidemiol,2002;30(4):241-7.

7. Vargas R, Herrera M. Estudio de prevalencia de caries en escolares de las comunidades rurales Mapuches de Panguinilague, Puquiñe y Lago Neltume. Provincia de Valdivia, X Región de Los Lagos. Rev Dent Chile, 2002;93(3):3-8.

8. Censo 2002 INE (Instituto Nacional de Estadísticas). http://www.ine.cl/ cd2002/index.php, accesado agosto 2009.

9. World Health Organization. Oral health survey. Basic methods. Geneva: World Health Organization,1997.

10. Caplan DJ, Weintraub JA. The oral health burden in the United States: a summary of recent epidemiologic studies. J Dent Educ1993; 57(12):853-62.

11. White BA, Caplan DJ, Weintraub JA. A quarter century of changes in oral health in the United States. J Dent Educ,1995;59(1):19-57.

12. Fure $\mathrm{S}$. Ten year incidence of tooth loss and dental caries in elderly Swedish individuals. Caries Res,2003;37(6):462-469.

13. Fure S, Zickert I. Incidence of tooth loss and dental caries in 60, 70 and 80 year old Swedish individuals. Community Dent Oral Epidemiol, 1997;25(2):137-42.

14. Wang HY, Petersen PE, Bian JY, Zhang BX. The second national survey of oral health status of children and adults in China. Int Dent $J$, 2002;52(4):283-90.

15. Madlena M, Hermann $P$, Jahn M, Fejerd P. Caries prevalence and tooth loos in elderly Chinese. Caries Res,2000;34(3):205-13.

16. Gamonal J. Tesis para optar al grado de Magíster en Periodontología. Facultad de Odontología, Universidad de Chile, 1996.

17. Urzua I. Tesis para optar al grado de Magíster en Ciencias Odontológicas con mención en Cariología. Facultad de Odontología, Universidad de Chile, 2009.

18. Rihs LB, da Silva DD, de Sousa Mda L. Dental caries and tooth loss in adults in a brasilian state. J Appl Oral Sci,2009;17(5):392-6.

19. Truin $G$, Koning $\mathrm{K}$, Kalsbeer $\mathrm{H}$. Trends in dental caries in the Netherlands. Adv Dent Res, 1993;7(1):15-18.

20 Downer M. Changing trends in dental caries experience in Great Britain. Adv Dent Res,1993;7(1):19-24.

21. Brennan DS, Spenser AJ. Changes in caries experience among Australian public dental patients between 1995/96 and 2001/02. Aust Public Heath,2004;28(6):542-548.

22. Bourgeois D, Nihtila A, Mersel A. Bull World Health Organ,1998;76 (49:413-417).

23. Steele A. Clinical and behavioural risk indicators for root caries in older people. Gerodontology,2001;18:95-101.

24. Ministerio de Salud, Chile. Resultados I Encuesta Nacional de Salud Chile 2003. http://epi.minsal.cl/epi/html/invest/ENS/ENS.htm. Accesado Agosto 2009. 